\title{
Variable Stars and Data-Intensive Astronomy
}

\author{
Nikolay N. Samus ${ }^{1,2}$ and Sergey V. Antipin ${ }^{2,1}$ \\ ${ }^{1}$ Institute of Astronomy (Russian Academy of Sciences), 48, Pyatnitskaya Str., Moscow \\ 119017, Russia; email: samus@sai.msu.ru \\ ${ }^{2}$ Sternberg Astronomical Institute, M.V. Lomonosov Moscow State University, 13, University \\ Ave., Moscow 119991, Russia; email: antipin@sai.msu.ru
}

\begin{abstract}
Extensive discoveries of variable stars in ground-based photometric surveys and in observations from space missions that provide unprecedented accuracy demonstrate that, till the most recent time, we knew only a tiny fraction of all detectable variable stars of our Galaxy. As a result, our knowledge on stellar variability types, related physical processes, variable-star statistics turn out to be based on an unrepresentative sample and are expected to be radically revised in the near future. The flow of new discoveries results in quite difficult problems for catalogs of variable stars, making it impossible to proceed in their compilation in the traditional way. Regretfully, automatic solutions are still not completely satisfying. Though not able to suggest a perfect way out, we present our vision of the future of variable-star research.
\end{abstract}

Keywords. catalogs, stars: statistics

\section{Introduction}

Since 1940s, the Moscow team of variable-star researchers is working, on behalf of the IAU, on catalogs of variable stars. This work encounters unprecedented difficulties in the era of data-intensive astronomy.

Probably the most fundamental problem is that there is no generally accepted brightnessvariation amplitude limit the star must exceed to be considered variable. The General Catalogue of Variable Stars (GCVS; Samus et al. 2012) currently contains stars with peak-to-peak amplitudes from $>19^{m}$ (V1500 Cyg) down to 0.004 ${ }^{m}$ ( $\alpha$ Aql). The number of known variables drastically increases with development of observing techniques.

\section{Current Statistics and Future Prospects}

The currently largest stellar catalog, USNO-B1.0 (Monet et al. 2003), contains about 1 billion stars down to $20-21^{m}$. Experience shows that one of 80-100 stars varies at the $0.03-0.05^{m}$ level, and thus some 10 million stars are potentially detectable as variables with a ground-based 1-m telescope, ordinary CCD detector, and standard software for automatic search for variable stars. The largest modern lists of known galactic variable stars (like VSX, Watson et al. 2007, supported by the AAVSO) contain about 200000 objects; it means that we know no more than $2 \%$ of all detectable variables of the Galaxy, a percentage lower than that of barion matter in the Universe! New discoveries can seriously change our ideas concerning frequency of different variability types. Even information contained in astronomical plate archives, if extracted and analyzed in digital form, changes statistics of variability types and period distributions (cf. Kolesnikova et al. 2010). Surveys performed with very small or moderate-sized CCD telescopes, like ASAS-3 (Pojmanski 1997), ROTSE-I/NSVS (Woźniak et al. 2004), SuperWASP (Pollacco et al. 
2006), Catalina (Drake et al. 2009), and others result in thousands of new variable-star discoveries, made by the survey teams and, mainly, by the astronomical community via data mining. Some of these surveys present their own lists of variable-star discoveries. It is difficult even to simply merge them with the GCVS because of different standards of positional accuracy and different level of detail of variable-star classification schemes.

If we turn to observations from outer space, amplitudes as low as $0.0001^{m}$ become detectable. According to results of just one month from the Kepler mission, about 60,000 periodic variables and 34,000 marginally periodic variables could be detected among some 150,000 targets (Basri et al. 2011). Such statistics is clearly incompatible with a concept of variable-star catalogs different from star catalogs in a more general sense; it makes impossible the traditional star-by star basis (with human expertise) our team uses, for decades, in our GCVS work.

Catalogs of variable stars contain classification of variables according to the existing scheme. The GCVS system clearly needs improvement; however, it seems too early to introduce a new scheme since dramatic changes are expected in the near future. Until recently, there were no automatic classification systems permitting to find the star's type from photometric data (see, however, Blomme et al. 2011 for a rather promising new development). Unfortunately, photometry alone is often insufficient for variable-star classification, additional (spectroscopic, X-ray, kinematical) information can be needed. The problem of really effective automatic classification needs further efforts to solve it.

\section{Brief Conclusions}

With full-scale results from Kepler (and then from similar missions) approaching, the number of known variable stars will drastically increase and everything we know about variability statistics will have to be revised. We are forced to admit that the era of traditional variable-star catalogs is probably near its end, despite traditional Argelanderstyle variable-star names being still popular.

Perhaps the only possible solution for the predictable future could be compilation of universal star catalogs, with variability information contained in them as a minor but important part.

\section{Acknowledgements}

We thank all participants of the GCVS team for their many-year effort aimed at high-quality catalogs of variable stars. Our research is supported by the Program "NonStationary Processes of Objects in the Universe" of the Presidium of Russian Academy of Sciences and by the Russian Foundation for Basic Research (grant 11-02-00495).

\section{References}

Basri, G., Walkowicz, L. M., Batalha, N., et al. 2011, AJ, 141, 20

Blomme, J., Sarro, L. M., O’Donovan, F. T., et al. 2011, MNRAS, 418, 96

Drake, A. J., Djorgovski, S. J., Mahabal, A., et al. 2009, ApJ, 696, 870

Kolesnikova, D. M., Sat, L. A., Sokolovsky, K. V., et al. 2010, ARep, 54, 1000

Monet, D. G., Levine, S. E., Canzian, B., et al. 2003, AJ 125, 984

Pojmanski, G. 1997, AcA, 47, 467

Pollacco, D., Skillen, I., Cameron, A. C., et al. 2006, PASP, 118, 1407

Samus, N. N., Durlevich, O. V., Kazarovets, E. V., et al. 2012, General Catalogue of Variable Stars (GCVS Database, Version 2012 Jan.), CDS B/gcvs

Watson, C. L., Henden, A. A., \& Price, A. 2007, JAAVSO 35, 414

Woźniak, P. R., Vestrand, W. T., Akerlof, C. W., et al. 2004, AJ, 127, 2436 